\title{
Lane Formation in Driven Binary Complex Plasmas on the International Space Station
}

\author{
K. R. Sütterlin, H. M. Thomas, \\ A. V. Ivlev, G. E. Morfill \\ Max Planck Institute \\ for Extraterrestrial Physics \\ 85741 Garching, Germany
}

\author{
V. E. Fortov, A. M. Lipaev, \\ V. I. Molotkov, O. F. Petrov \\ Joint Institute for High Temperatures \\ 125412 Moscow, Russia
}

\author{
A. Wysocki, H. Löwen \\ Heinrich-Heine-Universität \\ Düsseldorf \\ 40225 Düsseldorf, Germany
}

\begin{abstract}
We conducted a series of experiments to study lane formation in complex plasma, using the PK-3 Plus laboratory on the International Space Station (ISS). In our experiments small (driven) microparticles penetrate into a cloud of big (background) particles, revealing a strong tendency towards lane formation: When the driving force is strong enough, particles of both sorts form interpenetrating lines. Typically, the lanes exhibit a considerable anisotropic structural order accompanied by an enhancement of their (unidirectional) mobility. Laning is quantified locally in terms of the anisotropic scaling index. Using a "nematic" order parameter the observed time-resolved laneformation process is compared to a set of computer simulations of a binary Yukawa model with Langevin dynamics. This yields not only a perfect fit, but also reveals a strong influence of the initial experimental conditions on the dynamics of lane formation.
\end{abstract}

\section{INTRODUCTION}

The laboratory PK-3 Plus [1] on board the International Space Station (ISS) allows the research of complex plasmas under microgravity conditions. This research complements the work on the ground and is mandatory for certain precision experiments. PK-3 Plus is the second generation laboratory on the ISS already. Compared to its pre-cursor PKE-Nefedov it has an advanced hard- and software. Much better diagnostics and especially a much better homogeneity of the complex plasma allow more detailed investigations. Figure 1 shows a schematic view of the experimental setup. PK-3 Plus offers a wide variety of parameters. Argon and Neon can be used to produce an rf discharge. Neutral gas pressure, i.e. damping, as well as discharge power can be changed by two orders of magnitude. Particles of six different sizes, from 1.55 to $14.9 \mu \mathrm{m}$ in diameter, can be injected to form a complex plasma. All these factors are used to tune the details of (inter)particle dynamics. The possibility to resolve single particle motion in complex plasma experiments allows fully kinetic analysis.

One investigation, which uses these special features, is lane formation. This phenomenon can be found everywhere in nature when two species of particles are driven against each other. It is a genuine nonequilibrium transition [2] which depends on the details of the particle interactions and their dynamics [3], and is of considerable interest in different

\footnotetext{
${ }^{0}$ Manuscript received July 27, 2009. This work was supported by DLR/BMWi (grant no. 50WP0203), DFG through the SFB TR6, and RFBR (grant no. 08-02-00444).
}

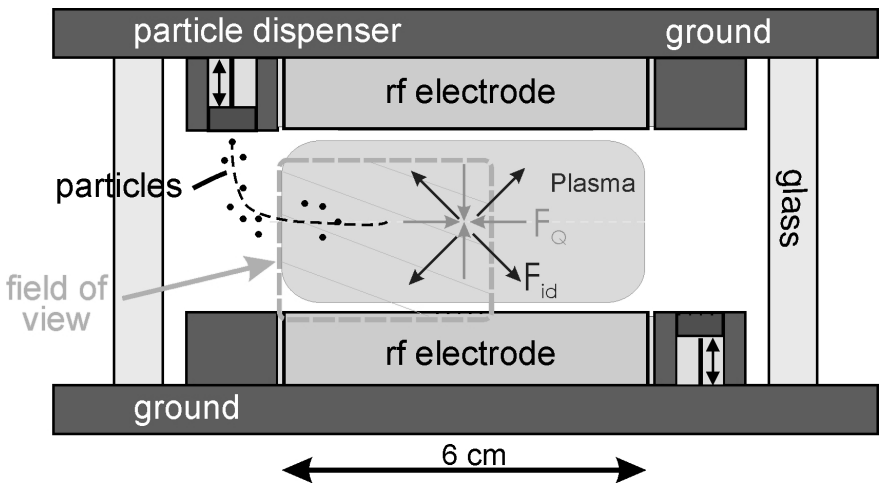

Fig. 1. 2D sketch of the PK-3 Plus setup. Dashed rectangular box indicates the field of view (see fig. 2).

branches of physics. Lane formation is most commonly known from pedestrian dynamics in highly populated pedestrian zones [4], but also occurs in different systems of driven particles, such as colloidal dispersions [5]-[7], lattice gases [2], and molecular ions [8]. Particle laning in complex plasmas [9] occupies the important intermediate dynamical regime between undamped fluids and fully damped colloidal suspensions [10], [11]: the "atomistic" dynamics associated with the interparticle interaction is virtually undamped whereas the large-scale hydrodynamics is determined by friction.

While steady-state lanes have been studied in detail, the dynamical pathway towards the laning is still under debate. PK3 Plus offers the perfect system to study the dynamics of lane formation [12]. Under microgravity conditions the dominant forces (see Figure 1) are the electrostatic force $F_{Q}$ arising from an electric potential with a maximum in the centre, and the ion drag force $F_{i d}$. The latter is due to the drift of positive ions along the electric field out from the centre and the resulting momentum transfer to the particles. These forces are all size dependent. The net force (pointing outward) increases with the particle radius. Thus the equilibrium position of smaller particles is closer to the centre than that of bigger ones.

For laning experiments we start by filling almost the whole chamber with big particles that form a stable background. Then smaller particles are injected from outside. The net force pulls the small particles through the cloud of big particles 


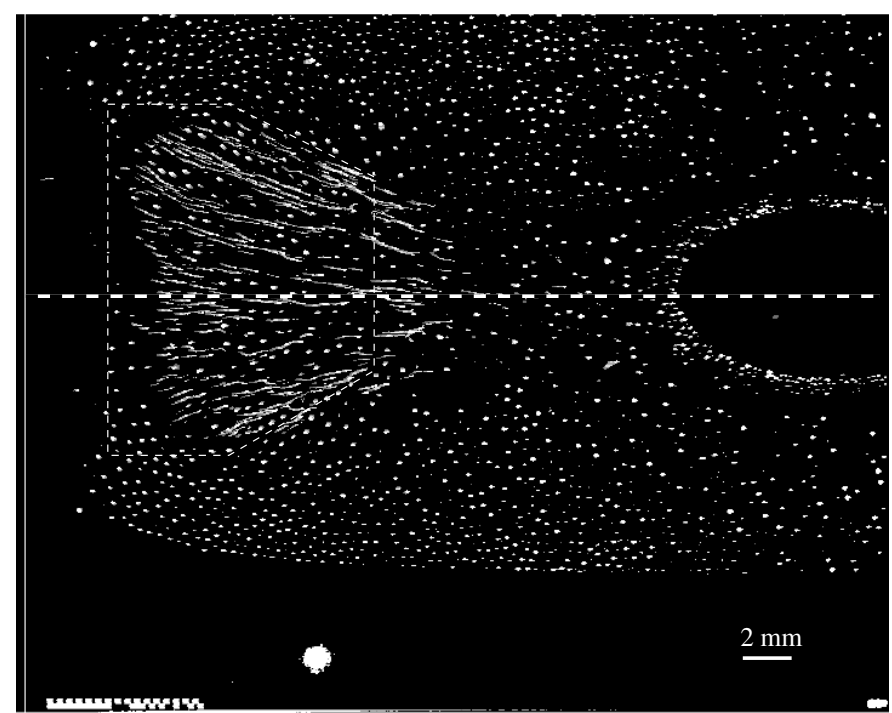

Fig. 2. Lane formation in complex plasmas. A short burst of small $(3.4 \mu \mathrm{m})$ particles is injected into a cloud of big $(9.2 \mu \mathrm{m})$ background particles. Small particles are driven towards the center, forming lanes. The particles are illuminated by a thin laser sheet of $\simeq 0.35 \mathrm{~mm}$. The picture is a superposition of consecutive images: the fast small particles can be identified as long tracks, the inert big particles look like points. One can clearly see, that also the big particles are arranged into lanes. The dashed line indicates the midplane of the PK-3 Plus chamber, the dashed box marks the measurement area for lane formation of big particles (see sec. IV-A1).

towards the center of the chamber. Under certain conditions this penetration appears in lanes, in order to avoid mutual collisions. A series of dedicated experiments was carried out on the ISS. These involved various combinations of "big" and "small" monodisperse particles $(2.55,6.8,9.2$, and $14.9 \mu \mathrm{m}$ diameter for "big", and $1.55,2.55$, and $3.4 \mu \mathrm{m}$ for "small"), with different neutral gases and pressures (argon between 10 and $60 \mathrm{~Pa}$ and neon at $60 \mathrm{~Pa}$, to control the friction rate), and for different $\mathrm{rf}$ discharge powers (to control the screening length and accordingly the particle interaction).

\section{EXPERIMENTS}

In this paper we analyze experiments at a pressure of $30 \mathrm{~Pa}$ using a background complex plasma formed by "big" $9.2 \mu \mathrm{m}$ diameter particles. Into this a single short burst of "small" particles with $3.4 \mu \mathrm{m}$ diameter is injected in plane with the illumination. Experimental conditions keep the background in a liquid state. In addition, very slow large-scale vortex motion is constantly mixing the background particles [13].

The particles are illuminated by a thin laser sheet of $\simeq$ $0.35 \mathrm{~mm}^{1}$. The scattered light is recorded under $90^{\circ}$ using a $50 \mathrm{~Hz}$ PAL progressive scan CCD camera. Figure 2 is an overlay of consecutive frames highlighting the lanes formed by small particles while they penetrate the cloud of big particles. Closer inspection reveals that the mostly inert big particles are combed into lanes, too. Thereby small and big particles from

\footnotetext{
${ }^{1}$ We see just a thin layer of particles around the center of the chamber. There are 3 to 4 thousand illuminated particles in the field of view, while the whole system consists of about half a million of particles.
}

an array of interpenetrating strings. After the small particles passed by the strings formed by big particles slowly dissolve.

For a full kinetic analysis of the development of lanes the trajectories of all visible particles were extracted from the images. This is especially difficult for the small particles: First they scatter less light than the big particles. Second they are quite fast, especially before entering the background, leaving long (small particles travel inter-particle distances of the background cloud per frame) and very faint traces on the images. Third the small particles are scattered violently in collisions with big particles, often out of the illuminated volume. Standard tracking algorithms, that first find objects in single frames, and then try to identify objects in consecutive frames are unable to track the small particles. For this paper we had to develop a different tracking algorithm. Particles are identified by the complete light curve they leave in the movie. These light curves are processed carefully, to make sure that one curve is produced by exactly one particle. To these light curves a simple model - twice differentiable curves - of particle motion is fitted to extract particle position, velocity and acceleration at each time step.

After extracting the full kinetic information, particles are sorted by their velocities into two groups. Particles moving towards the center with large enough velocity are considered to be small. All other particles are considered to be big. This proves to be a very good selection algorithm providing no false positives, i.e. big particles wrongly identified as small. Unfortunately this criterion results in $30 \%$ false negatives, i.e. small particles wrongly identified as big.

\section{DATA ANALYSIS}

To quantify lane formation in terms of nonequilibrium phase transitions we need a suitable order parameter. The local anisotropy of lanes lends itself to using the anisotropic scaling index method [14]. On top of this local measure we define a global "nematic" order parameter. To calibrate our measures and to get some insight into experimental parameters that are not directly measurable we resort to numerical simulations.

\section{A. Anisotropic scaling index}

The scaling index $\alpha=\partial \log \rho\left(\mathbf{r}_{i}, R\right) / \partial \log R$ is the logarithmic derivative of a local density $\rho\left(\mathbf{r}_{i}, R\right)=$ $\sum_{j=1}^{N} s\left(d_{i j} / R\right)$, for a set of points $\left\{\mathbf{r}_{i}\right\}, i=1, \ldots N . s$ being a certain shaping function and $d_{i j}=\left|\mathbf{r}_{i}-\mathbf{r}_{j}\right|$. We use $s=e^{-\left(d_{i j} / R\right)^{2}}$ which gives

$$
\alpha\left(\mathbf{r}_{i}, R\right)=\frac{2 \sum_{j=1}^{N}\left(d_{i j} / R\right)^{2} e^{-\left(d_{i j} / R\right)^{2}}}{\sum_{j=1}^{N} e^{-\left(d_{i j} / R\right)^{2}}} .
$$

$\alpha\left(\mathbf{r}_{i}, R\right)$ determines the dimensionality of the point distribution around $\mathbf{r}_{i}$, in a vicinity defined by the scale $R$. For example, $\alpha\left(\mathbf{r}_{i}, R\right) \simeq 1$ means that the local structure is close to a straight line. The spatial scale $R$ is an important mesoscopic measure of the local environment. In the range of relevant scales $\alpha$ is practically independent of $R$.

To define the anisotropic scaling index, we use a "stretch metric" for the distance measure $d_{i j}$ : In 2D the metric is 
determined by $\epsilon(>1)$, the relative stretching of two principal axes, and the direction of stretching $\mathbf{u}=(\cos \theta, \sin \theta)$. The anisotropic scaling index $\alpha\left(\mathbf{r}_{i}, R, \epsilon, \theta\right)$, is derived directly from Eq. (1).

\section{B. "Nematic" order parameter}

Using the anisotropic scaling index method we associate each point $\mathbf{r}_{i}$ with the unit vector $\mathbf{u}_{i}$ in the "preferred" direction of the local anisotropy, as determined by the angle $\theta_{i}$ at which the "anisotropic contrast" $\alpha\left(\mathbf{r}_{i}, R, \epsilon, \theta_{i}+\pi / 2\right)-$ $\alpha\left(\mathbf{r}_{i}, R, \epsilon, \theta_{i}\right)$ is maximized ${ }^{2}$. For each point we define a corresponding rod with the direction $\mathbf{u}_{i}$.

The global laning on a 2D plane can be characterized with the second-rank tensor $Q_{\alpha \beta}=2 N^{-1} \sum_{i=1}^{N} \mathbf{u}_{i} \otimes \mathbf{u}_{i}-\delta_{\alpha \beta}$, analogous to that used to quantify order of the nematic phase. The largest eigenvalue of $Q_{\alpha \beta}$ defines the laning order parameter $S$. With $S=1$ for a perfect alignment and $S=0$ for a disordered phase. The corresponding eigenvector ("nematic director") $\langle\mathbf{u}\rangle$ defines the direction of the global laning, and the global laning angle via $\cos \Theta=\langle\mathbf{u}\rangle \cdot \mathbf{e}_{x}$.

\section{Numeric simulations}

To complement our complex plasma experiments we used two independent numerical simulations. First a 2D simulation of the PK-3 Plus discharge chamber using a plasma fluid code (described in Ref. [15]), providing self-consistent coupling of dust species to the discharge plasma. This provided us with parameters of the discharge plasma, such as plasma density, electron temperature, and electric fields. For the background complex plasma this simulation also suggested that the cloud of big particles is "self-confined" at its edges due to the selfconsistent plasma field (cf. Fig. 13 of Ref. [15]) whereas inside the cloud the electric and ion drag forces practically compensate each other. For the small particles it predicted a nonzero net-force inside the background: for the experiment discussed here, a force of $f_{\mathrm{s}}=0.08 \mathrm{pN}$ pushing a small particle towards the center is predicted by the simulation.

Then we input these results (and measurements of previous experiments [16]) in a particle-resolved molecular dynamics (MD) simulation performed on the Langevin level [17] for a binary mixture of 5759 small and 12287 big particles (cf. Figure 3 ). Whereas the 2D plasma fluid code mainly provided us with experimental parameters that cannot be measured in PK-3 Plus onboard the ISS, the 3D MD simulation was used to calibrate our local structure measures and global order parameter. Unlike in the experiment we can directly control all parameters defining particle interaction individually and independently. Additionally initial conditions, especially relaxation time before the injection, can be varied. Comparing the measurements of different simulations allows us to identify the most important players in the dynamics of lane formation. The divergence from measurements of the experimental data allows us to separate the influence of coupled parameters,

\footnotetext{
${ }^{2}$ By definition the directions $\mathbf{u}_{i}$ and $-\mathbf{u}_{i}$ are equivalent, so that we consider the range $-\frac{\pi}{2} \leq \theta_{i} \leq \frac{\pi}{2}$.
}

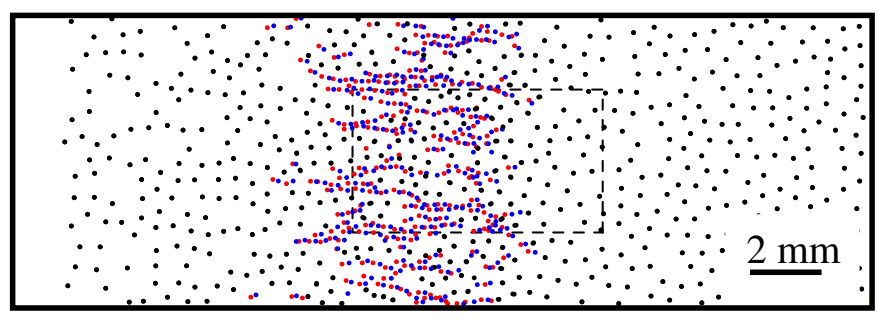

Fig. 3. Lane formation in MD simulation corresponding to the experiment shown in Fig. 2. The simulation is fully three dimensional. To get highest similarity between experimental and simulation data, the latter is cut in slices of $0.35 \mathrm{~mm}$ width, which are analyzed individually.

e.g. particle charge and screening length that define the interparticle potential.

\section{RESUlts}

To get the best fit numerical simulations we first put all measured variables of the experiment, geometries and all relevant physics into our simulations, and used theoretical estimates to get the most faithful representation of experimental conditions.

There are basically only two free parameters left to tune the simulation. 1) The decomposition of the coupling parameter $\Gamma$ into particle charge and screening length. 2) The initial state of the background complex plasma, into which the smaller particles are injected. In principle the initial state of the small particles could also have some influence. In our case, due to the much bigger inertia of the background particles, there is no influence, as all structural order in the cloud of injected small particles is completely lost upon impact.

First we compare the "best fit" simulations to the actual experimental data. Second, using the possibilities of numeric experiments, we investigate the influence of initial conditions on the dynamics of lane formation, and verify our choices for particle charge and screening length, which can not be measured directly in the experiment.

\section{A. Best fit of simulations and experiment}

As explained in sec. III-C we used measurements of previous experiments for the particle charge of small particles: $Q_{\mathrm{s}}=-4000 e$, and the charge of big particles is derived by the size ratio to be: $Q_{\mathrm{b}}=-11000 e$. The screening length is calculated from the plasma density as given by the plasma fluid code: $\lambda=150 \mu \mathrm{m}$.

1) Big particles: For the analysis of laning in the cloud of big particles a fixed measurement box (cf. figures 2 and 3) well within the cloud was used, both for experiments and simulations. This is necessary to remove edge effects of the scaling index method, and in the case of experiments, to remove unaffected parts of the cloud from statistics.

To analyze dynamics of laning in the background complex plasma using anisotropic scaling index we used a stretching of $\epsilon=5$, and a special scale $R=1200 \mu \mathrm{m}$.

A comparison of the temporal evolution of lanes in the background complex plasma between experiment and best fit simulations is given in figure 4 . The important features 


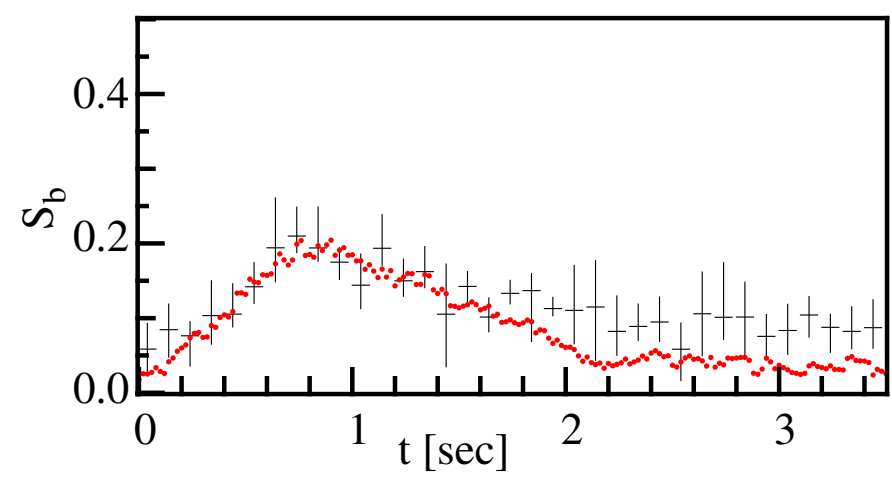

Fig. 4. Evolution of the "nematic" order parameter for big particles $S_{\mathrm{b}}$, as obtained from the anisotropic scaling index analysis of the experiment (crosses) and MD simulation (dots).

are the initial non zero value of the order parameter for the experimental data, the rise and fall times, the non vanishing "final" value of the order parameter, the continuous variation of the order parameter.

Initially there is no anisotropy in the simulation data, still the experiment already shows some non zero initial value of the order parameter. This results from a density gradient in the experimental data, that is not present in the simulation.

The rise time is basically identical to the mixing time, i.e. the time necessary for the small particles to completely fill the measurement box. That means that within the temporal resolution of the experiment lanes in the background complex plasma are formed "immediately", by the small particles streaming through it. This can also be confirmed visually, stream lines once formed do not evolve (or improve) while more small particles stream through.

The fall time consists of three parts. First the demixing time, needed for the last small particles to leave the measurement box. As small particle velocity is almost constant, this time is identical to the mixing time. One sees immediately that lanes in the background cloud persist after the small particles are gone. Second these lanes decay at a characteristic timescale of $\sim 1 \mathrm{~s}$ (which much slower than would be expected from the self-diffusion timescale $\left.\sim m_{\mathrm{b}} \nu_{\mathrm{b}} \Delta_{\mathrm{b}}^{2} / T\right)^{3}$. Third, after this fast relaxation $S_{\mathrm{b}}(t)$ stays on some intermediate plateau, indicating that the structural relaxation is not complete. Thus the process of complete relaxation might involve metastable states.

Therefore the results point at a continuous second order phase transition.

2) Small particles: Similar to the big particles above, we did not take all small particles into account for the analysis. Only small particles within the cloud of big particles are considered. This is necessary for the experimental data, as here small particles outside the cloud of big particles are either completely out of the field of view or much too fast to be detected in the movie. In figure 5 the evolution of $S_{\mathrm{s}}(t)$ is divided in two parts, the first being injection of the cloud

\footnotetext{
${ }^{3}$ With mass $m_{\mathrm{b}}$, friction constant $\nu_{\mathrm{b}}$, mean particle separation $\Delta_{\mathrm{b}}$ and thermal energy of the dust particles $T$.
}

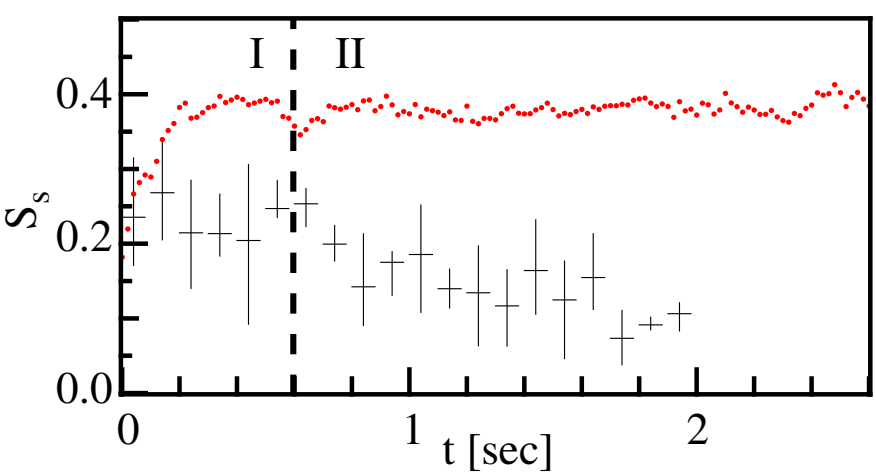

Fig. 5. Evolution of the "nematic" order parameter for small particles $S_{\mathrm{S}}$, as obtained from the anisotropic scaling index analysis of the experiment (crosses) and MD simulation (dots). Injection stage I and steady-state stage II are indicated. For the experiment we cannot analyze lanes for more than two seconds, because particles approach the center of the chamber where the driving force vanishes.

of small particles into the background where the number of particles taken into account is increasing, and the second part is the steady state when the small particles are all embedded within the cloud of big particles.

We used a stretching of $\epsilon=7$, and a special scale $R=2200 \mu \mathrm{m}$ for the detection of lanes formed by small particles. Unlike the big particles, the formation of lanes in small particles, i.e. $S_{\mathrm{s}}(t)$ (c.f. figure 5), has no rise or fall times. In addition the order parameter seems to indicate much lower structural ordering for experimental data than for the simulations (see sec. IV-B1).

The order parameter $S_{\mathrm{s}}$ has a high value from the very beginning, initially varying strongly due to statistical fluctuations as the number of particles analyzed is rising from zero to a few hundred.

This means, that small particles entering the cloud of big particles are immediately - i.e. order of the time resolution of the experiment or faster - sorted into lanes, or enter into channels created by earlier small particles. As they are very fast during initial impact, lanes are created within the first few inter particle distances of the background complex plasma.

\section{B. Optimizing simulation parameters}

1) Artificial Thinning: For experimental data the order parameter of small particles $S_{\mathrm{s}}$ is suppressed compared to the simulation data. This is mainly an effect of the dynamical cuts applied to the experimental data for selection of small particles. As explained in the description of the experiment (see sec. II) we lose a substantial amount of small particles from the analysis, which has a strong influence on the anisotropic scaling index of the particles and thus hinders efficient detection of lanes. Figure 6 shows the influence of random thinning of the simulation data, leading to a systematic decrease of the nematic order parameter.

2) Particle Charge: Not all parameters of the simulation could be fixed by measurements or derived with high accuracy from theory or simulation. Especially the particle charge and the screening length have substantial error margins. 


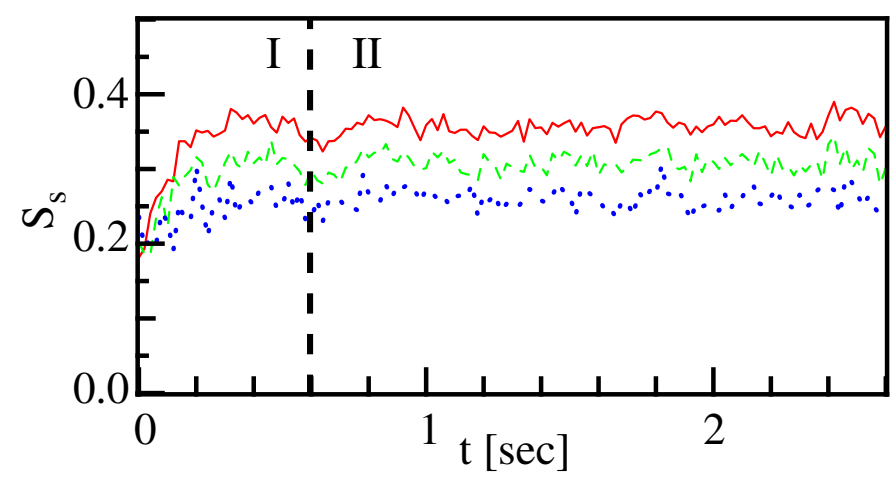

Fig. 6. Influence of random "thinning" of the simulation data on the value of $S_{\mathrm{s}}$. The order parameter is decreasing with increased thinning fraction: $10 \%$ (solid line), $30 \%$ (dashed), $50 \%$ (dotted).

In the MD numerical simulation, we can change particle charge and screening length individually and independently. For this paper we vary them in such a way, that the resulting coupling parameter $\Gamma$ stays constant: ${ }^{4}$ i.e. without changing the phase of the background. Thus changes in dynamical behavior indicate which set of parameters is closest to the experimental conditions. We consider three parameter sets:

- $Q^{0} \lambda^{0}$ : The "best fit", derived from measurements, using plasma parameters from the $2 \mathrm{~d}$ fluid code, and tuning the MD simulation to give the same kinematics, i.e. velocity distribution, as the experiment. Here small particles have a charge of $Q_{\mathrm{s}}^{0}=-4000 e$, big particles have $Q_{\mathrm{b}}^{0}=$ $-11000 e$, and the screening length $\lambda^{0}=150 \mu \mathrm{m}$. This is represented by a solid line in the plots of figure 7 .

- $Q^{+} \lambda^{-}$: Particle charge is increased $Q_{\mathrm{s}}^{+}=-8278 e$ and $Q_{\mathrm{b}}^{+}=-25100 e$. The screening length is decreased appropriately to $\lambda^{-}=100 \mu \mathrm{m}$. Plotted with dashed line.

- $Q^{-} \lambda^{+}$: Here particle charge is lower $Q_{\mathrm{s}}^{-}=-3000 e$ and $Q_{\mathrm{b}}^{-}=-8117 e$, whereas the screening length $\lambda^{+}=$ $182 \mu \mathrm{m}$ is longer. Represented by a dotted line.

There are two main differences: (i) The velocity distribution and mobility of the small particles within the cloud of big particles, and (ii) the characteristics of lane formation for small and big particles.

i) The mobility of small particles within the background cloud strongly depends on the dynamics of the big particles. Here $Q^{0} \lambda^{0}$ provides the highest mobility. Comparing mobility and velocity distribution of the three simulations to the experimental results, $Q^{0} \lambda^{0}$ represents the particle dynamics most faithfully, thus it is called "best fit".

ii) $Q^{0} \lambda^{0}$ is able to reproduce the dynamics of laning measured in the experiment fairly well, especially for the big particles.

In the case of $Q^{+} \lambda^{-}$the lanes formed by small particles during injection slowly decay while they propagate through the

${ }^{4}$ It is defined as $\Gamma=\frac{Q^{2}}{T \Delta} \mathrm{e}^{-\kappa}$, with particle charge $Q$, mean particle separation $\Delta=n^{-1 / 3}$ in terms of the particle number density $n$, lattice parameter $\kappa=\frac{\Delta}{\lambda}$, screening length $\lambda$ and thermal energy of the dust particles $T$.

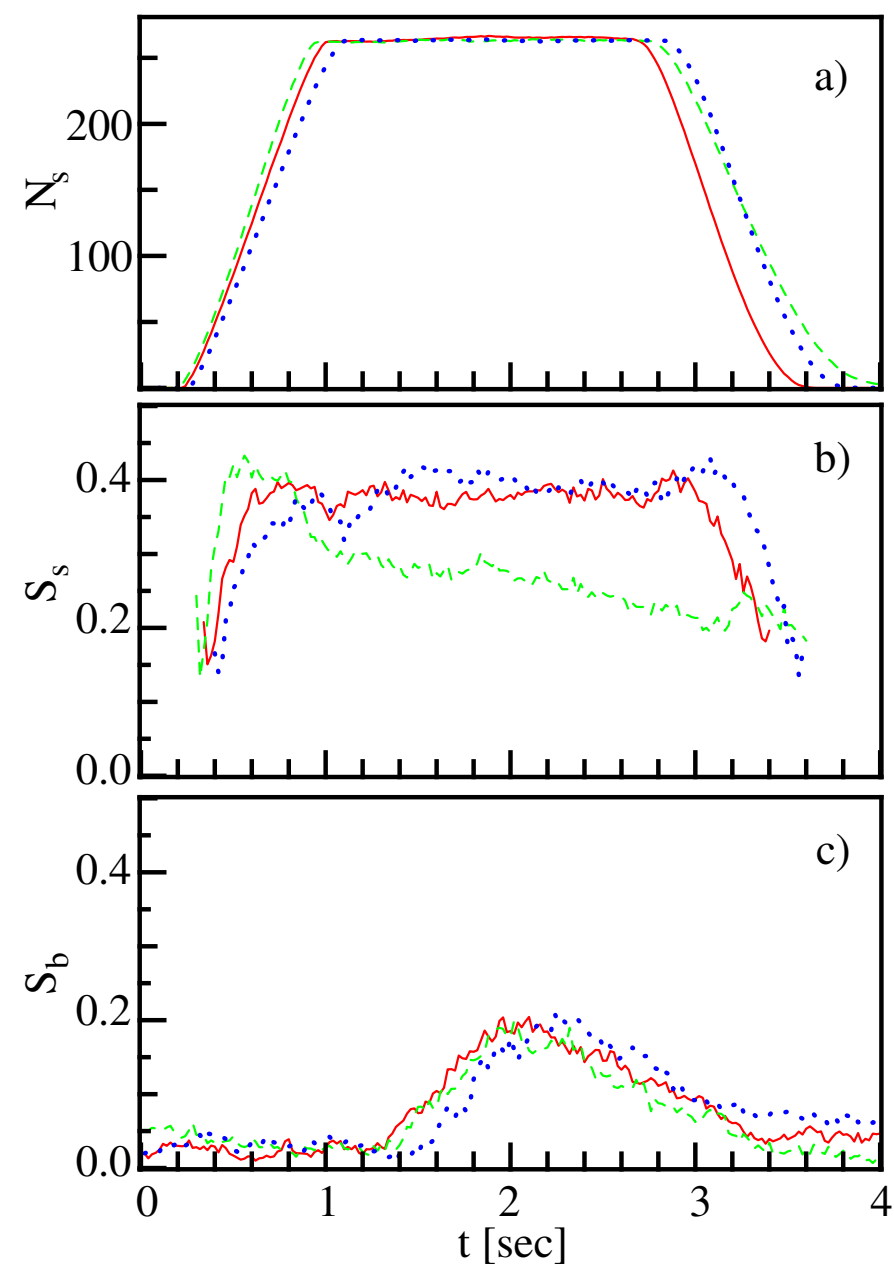

Fig. 7. Influence of particle charge and screening length on the dynamics of lane formation. The charge and screening length are varied in the MD simulations, so that the coupling parameter $\Gamma$ is kept constant: (solid) $Q^{0} \lambda^{0}$ : $Q_{\mathrm{s}}^{0}=-4000 e, Q_{\mathrm{b}}^{0}=-11000 e, \lambda^{0}=150 \mu \mathrm{m}$, (dashed) $Q^{+} \lambda^{-}$: $Q_{\mathrm{s}}^{+}=-8278 e, Q_{\mathrm{b}}^{+}=-25100 e, \lambda^{-}=100 \mu \mathrm{m}$, (dotted) $Q^{-} \lambda^{+}$: $Q_{\mathrm{s}}^{-}=-3000 e, Q_{\mathrm{b}}^{-}=-8117 e, \lambda^{+}=182 \mu \mathrm{m}$. Shown are a) the number of small particles inside the background cloud of big particles, the temporal evolution of the order parameter for b) small particles and c) big particles.

background cloud. The experimental data might hint to such a trend (see fig. 5) indicating that we could have underestimated particle charge or did not choose perfect coupling parameter. Yet the evolution of $S_{\mathrm{b}}$ is lacking the plateau.

This plateau and also all timescales of formation of lanes in big particles is very well reproduced by $Q^{-} \lambda^{+}$. Slightly better even than by the "best fit". For small particles this parameter set shows a slow increase of the order parameter during injection which is not at all supported by experimental data.

It is obvious, that dynamics of lane formation depend very sensitively on complex plasma properties. More variations of particle charge and coupling parameter must be investigated using MD simulations to better identify which aspects of dynamics of lane formation are influenced by specific parameters. 


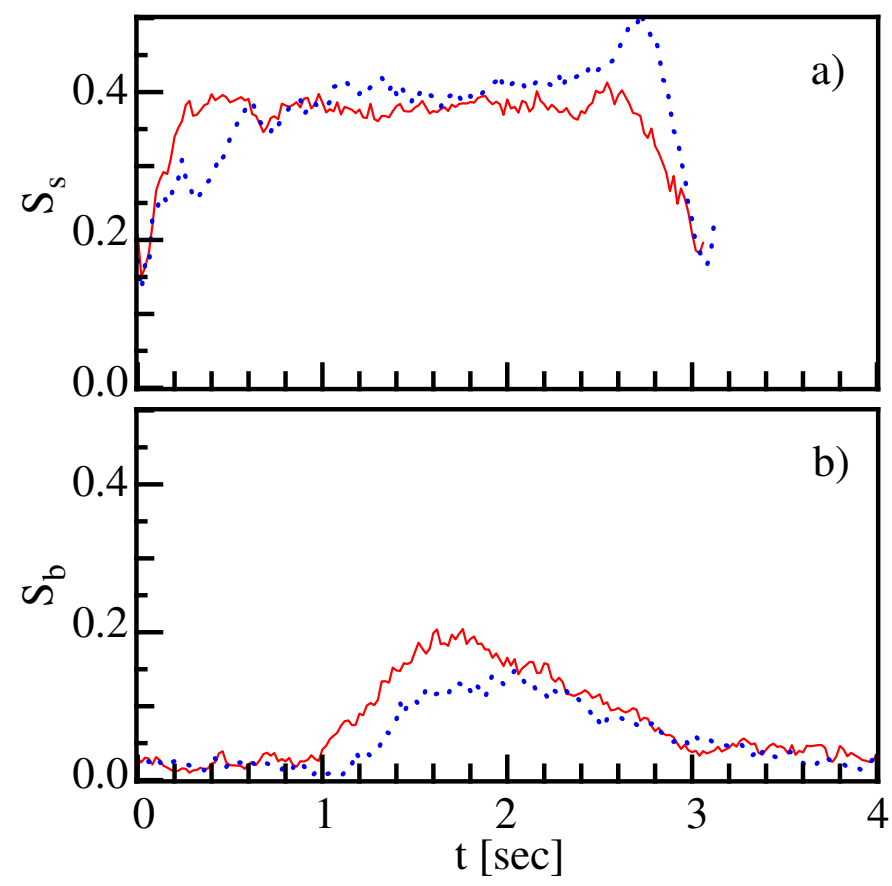

Fig. 8. Initial conditions influence lane formation. Small particles are injected into a background of big particles that has (solid line) just been thermally equilibrated for two seconds, as compared to (dots) an almost fully crystalline background. Shown is the temporal evolution of the laning order parameter for a) small, and b) big particles.

3) Initial Conditions: The main influence of initial conditions on the results is through the spacial configuration and phase state of the big particle cloud, before the small particle are injected.

For the best fit parameter set we let the numerical simulations equilibrate the background complex plasma for two seconds. This removes all initial heat, induced by the random initial positions and velocities, from the system. But the system still contains lots of internal energy and is far from a crystalline state. As far as we can tell this portrays the experimental conditions very well. We compare this configuration to numerical simulations that equilibrate the background for 1000 seconds. After such a long time the system is, except for the center, in a crystalline state, close to its minimal energy configuration. The big particles form layers parallel to the external confinement.

Figure 8 displays the main differences in dynamic behavior. First of all, in the case of an almost crystalline background, $S_{\mathrm{s}}$ rises slowly during the injection of small particles into the background of big particles, reaching its steady state only after all small particles are inside the background cloud. Also, as can be seen from fig. 8 b), the mobility of small particles is much lower, reaching the measurement area later. The big particles are much less influenced by the passage of small particles, resulting in a lower peak value of $S_{\mathrm{b}}$. Interestingly the decay of lanes in the background media is basically unmodified from what we see in the best fit dataset. Even though the big particles do not reach the same level of laning order, the processes of decay of local structure are not influenced much by the (initial) state or internal energy of the big particle cloud. Still it seems, that there is no - or a much lower - plateau in the decay of big particle lanes for the crystalline background. (This might be, because equilibration of local order is not going to a generic liquid state, but to an already quite well defined layered crystalline structure.) The peak in $S_{\mathrm{s}}(t)$ evolution (at about $t=2.6 \mathrm{~s}$ ), when the small particles leave the cloud of big particles, indicates that the later batches of small particles are better aligned than the forerunners (which would be in agreement with the difficulty of forming lanes during injection).

\section{CONCLUSION AND OUTLOOK}

Binary complex plasmas provide us with new insights into the dynamical regime of laning - between classic undamped fluids and fully damped colloidal suspensions. By combining the experimental studies and the particle-resolved Langevin simulations, we investigated the dynamical onset of lane formation in driven complex plasmas. The use of a very sensitive order parameter proved valuable in studying the onset of this non-equilibrium phase transitions, and in distinguishing influence of different complex plasma parameters on the dynamics of lane formation. We will continue to analyze more experiments and perform full parameter scan using MD simulations, especially to investigate laning in periodically driven [18] and crystalline [19] systems.

\section{REFERENCES}

[1] H. M. Thomas et al., New J. Phys. 10, 033036 (2008).

[2] B. Schmittmann and R. K. P. Zia, Phys. Rep. 301, 45 (1998).

[3] M. Rex and H. Löwen, Eur. Phys. J. E 26, 143 (2008).

[4] D. Helbing, I. J. Farkas and T. Vicsek, Phys. Rev. Lett. 84, 1240 (2000).

[5] J. Dzubiella, G. P. Hoffmann, and H. Löwen, Phys. Rev. E 65, 021402 (2002).

[6] M. E. Leunissen et al., Nature 437, 7056 (2005).

[7] C. Reichhardt and C. J. Olson Reichhardt, Phys. Rev. E 74, 011403 (2006).

[8] R. R. Netz, Europhys. Lett. 63, 616 (2003).

[9] G. E. Morfill et al., New J. Phys. 8, 7 (2006).

[10] V. E. Fortov et al., Phys. Rep. 421, 1 (2005).

[11] P. K. Shukla and A. A. Mamun, Introduction to Dusty Plasma Physics (IOP, Bristol, 2001).

[12] K. R. Sütterlin, A. Wysocki, A. V. Ivlev, C. Räth, H. M. Thomas, M. Rubin-Zuzic, W. J. Goedheer, V. E. Fortov, A. M. Lipaev, V. I. Molotkov, O. F. Petrov, G. E. Morfill, H. Löwen, Phys. Rev. Lett. 102, 149901 (E) (2009)

[13] M. Rubin-Zuzic et al., New J. Phys. 9, 39 (2007).

[14] C. Räth et al., Mon. Not. R. Astron. Soc. 337, 413 (2002).

[15] V. Land and W. J. Goedheer, New J. Phys. 8, 8 (2006).

[16] S. V. Annibaldi et al., New J. Phys. 9, 327 (2007).

[17] A. V. Ivlev et al., Phys. Plasmas 12, 092104 (2005).

[18] L. Corte et al., Nature Phys. 4, 420 (2008).

[19] Y. Roichman, V. Wong, and D. G. Grier, Phys. Rev. E 75, 011407 (2007). 